\title{
Effects of a transphenomenal parameter on the visual perception of form'
}

\author{
EARL A. ALLUISI, UNIVERSITY OF LOUISVIL LE \\ THOMAS J. HALL, LOCKHEED-GEORGIA COMPANY
}

\begin{abstract}
Abstraet
A transphenomenal parameter, based on the use of metric figures selected with a constrained sampling rule rather than a random rule, was found to increase response time significantly in a paper-and-pencil figure-cancellation task. Response time also increased with increasing figure complexity, but was unaffected by changes in the size of the figure. Comparison of these results with those of a prior study that differed only in terms of the choice situation presented S suggests that perceptual performance may be affected by an interaction of task variables with the physical parameters of form.
\end{abstract}

\section{Problem}

In their recent review of the literature pertaining to the visual perception of form Michels \& Zusne (1965) classify the physical parameters of form into three major types. Changes in the first type of parameter affect both the uncertainty of the figure (in an informational sense) and its specific structure or shape. Changes in the second type affect only the structure or shape of the figure and leave unaffected its uncertainty. Changes in the third type of parameter affect neither the uncertainty nor the shape of the figure (e.g., "transpositional" parameters such as area or size).

There remains, of course, a fourth logical class; namely, that of the "transphenomenal" parameters, changes in which affect only the uncertainty of the figure and not its specific structure or shape. Evidence for the empirical validity of this logically meaningful class has appeared in the literature (cf. Fitts \& Leonard, 1957; Baker \& Alluisi, 1962), but without stress of this point. Indeed, some of the evidence has appeared in entirely different settings, as in the measurement of contextual, cognitive, or situational effects on perceptual performance (e.g., Fitts \& Switzer, 1962).

Direct evidence of the effects of a transphenomenal parameter-the use of Redundancy-I or constrained metric figures rather than random metric figures-is provided in the present study where parameters of all four types are used in a paper-and-pencil figure-cancellation task.

Method

The shapes used are relatively simple; they have the appearance of bar graphs, solidly dark on a white background. They are called "metric figures," and have been described in detail elsewhere (Fitts \& Leonard, 1957; Baker \& Alluisi, 1962). They are constructed on the basis of an assumed underlying matrix of cells that defines a population of such figures from which specific figures can be sampled. Thus, if figures are constructed by random sampling of column heights, a 4 by 4 cell matrix would define a population of $4^{4}$ or 256 random 4 -column metric figures; an 8 by 8 matrix would define a population of $8^{8}$ or $16,777,216$ random 8 -column metric figures. Were the random sampling of column heights constrained by the requirement that each possible column height appear once and only once in each figure, populations of "constrained" or "Redundancy-I" figures would be defined-populations that are in fact subpopulations of the respective random-figure populations. The 4 by 4 matrix would thereby define a population of 4 ! or 24 constrained metric figures, as contrasted with 8 ! or 40,320 constrained metric figures in the population defined with the 8 by 8 matrix.

The use of matrices of different numbers of cells permits the definition of populations of figures of different complexities (i.e., different numbers of columns, column heights, and population sizes). When an equiprobable random metric figure from a population generated by a 4 by 4 matrix is identified, the uncertainty reduction associated with its identification is $\log _{2} 256$, or 8 bits. Under similar circumstances, identification of an 8-column figure is associated with 24 bits. If the figures were Redundancy-I rather than random metric figures, the corresponding uncertainty reduction associated with an identification would be 4.6 bits and 15.3 bits, respectively. It is in this sense that the use of constrained or Redundancy-I metric figures constitutes use of a transphenomenal parameter-the uncertainty reduction per figure identification is changed relative random metric figures, but the basic configuration of a 4 by 4 or 8 by 8 figure is unaffected.

Six samples of eight different figures were drawn, three each from populations of random and constrained (Redundancy-I) metric figures. One sample of each type (random or constrained) was drawn from a population generated by an 8 by 8 matrix; the other two samples were drawn from a 4 by 4 population.

In order to test for any possible effects of variation in the size of the detail of the figure or in the over-all area of the figure, each of the two samples of 4 by 4 figures differed in size. In one of the samples, the basic cell size was equal to that used in the 8 by 8 figures (approximately $0.04 \mathrm{~cm}$ sq.) and, therefore, the over-all size of the figure was $1 / 4$ the area of the 8 by 8 figure. In the other 4 by 4 sample, the cell size was increased (to about $0.16 \mathrm{~cm} \mathrm{sq.)} \mathrm{so}$ that the figure was equal in area to the 8 by 8 figures.

The stimulus figures were constructed by using small solid squares produced by a special key on an electric typewriter. The figures were then photographed and reproduced by off-set printing on $8-1 / 2$ by 11 -in white paper. The eight sampled figures of a given type appeared as target figures on each of eight different sheets that were then stapled together to form a subtest. The order of pages was balanced across the subtests, whereas the order of target figures and correct responses was balanced within each subtest. Six subtests, one for each of the six samples of figures (or experimental conditions), were stapled to form a test booklet, their order's being balanced across the tests.

Each sheet consisted of eight rows of figures. Each row consisted of a target figure on the left and three choice figures on the right: $31 / 2$ figure widths separated left from right, and $1 / 2$ figure widths separated both the choice figures and the rows.

The first (left-most) and second choice figures were metric figures of the same type as the target figure, whereas the third choice figure was in all cases an open square equal in over-all area to the cell matrix. The task required $\mathrm{S}$ to look at the target figure on the left of each row and then to cross out either the first or the second choice figure if it was identical to the target, or the open square if neither of the first two choice figures matched the target. Each completed test booklet consisted of eight cancellation responses for each of the 48 target figures, or 384 responses in all. 
The tests were administered to 69 introductory psychology students at Emory University; there were 31 males and 38 females. A 12-in diameter Telechron clock with sweep-second hand was placed at the front of the group-testing room so as to be visible clearly to all Ss. The clock was set to zero and was not started until actual testing began. Clock time to the nearest second was recorded by each $\mathrm{S}$ at the bottom of each page of the testbooklet as he completed that page.

\section{Results}

The mean time per response and the mean percentage of errors were used as criteria of performance, but no formal analysis of the latter was computed because so few errors were made. The mean percentages of incorrect cancellations made in responding to the three samples ranged from 1.08 to 3.88 with the random metric figures and 2.33 to 3.45 with the constrained figures.

The mean response times per figure cancellation (in seconds) were $2.67,2.74$, and 3.30 for the small and large 4 by 4 , and the 8 by 8 random metric figures, respectively, and $3.18,3.20$, and 4.39 for the small and large 4 by 4 , and the 8 by 8 constrained (Redundancy-I) figures, respectively. The results of an analysis of variance indicated that the differences among the six experimental conditions were statistically significant $(F=68.65, d f=5 / 340, p<.001)$ 。

Further partitioning by orthogonal components indicated that the difference between random and constrained metric figures was significant $(F=127.04$, $\mathrm{df}=1 / 340, \mathrm{p}<.001$ ), as was also the difference between the 4 by 4 and 8 by 8 complexities $(F=193.40, d f=1 / 340$, $\mathrm{p}<.001)$ and the interaction of these two effects $(F=22.37, d f=1 / 340, p<.001)$. Neither the difference between the small and large 4 by 4 figures, nor the interaction of this with use of random and constrained figures reached statistical significance (in each case the F-ratio was less than unity)。

\section{Diseussion}

The results of this study confirm and extend those of prior investigations (Fitts \& Leonard, 1957; Baker \& Alluisi, 1962) in demonstrating that the time required to identify a figure increases with increasing figure complexity and with use of constrained rather than random metric figures, whereas size of figure has no affect within the limits of size differences employed. Of the four types of parameters discussed in the introduction, complexity belongs to the first, size to the third, and the use of constrained metric figures to the fourth. No parameter of the second type was used in a way that would have permitted its evaluation, but rather the different figures (shapes) of a given com- plexity were employed as random variables to permit the replications necessary for a meaningful figurecancellation task.

The conditions of the present study differ in only two potentially important ways from the conditions of one of the eirlier investiga ons (see Experiment I in Baker \& Alluisi, 1962, Pp. 164-167)。First, 69 Ss were used in the present study, whereas 96 we1 used in the earlier one. This difference seems to be unimportant since the evidence indicates that the present study is at least as powerful as the previous one.

Secondly, the conditions of choice differed in the two studies. In the present case, $\mathrm{S}$ was shown eight different targets per page and for each target he had to indicate whether one or the other or neither of two choice figures matched the target figure. In the previous study, $S$ was shown one target figure at the top of a page that contained six rows of eight choice figures per row; also, one of the choice figures in each row always matched the target figure.

Thus, the studies differed in providing either a 3or an 8-choice situation, with or without changing targets for each choice, and with or without a matching figure's being always among the choice figures. The results of the two studies agree rather closely in all substantive respects except one: in the present study a significant interaction of type (random versus constrained) and complexity ( 4 by 4 versus 8 by 8 ) of figure was found to affect performance, whereas no such interaction was indicated in the prior study. This suggests that task variables (such as the choice situation) interact with the physical parameters of form in determining perceptual performance and that the generalizations of perceptual studies may have to be appropriately limited.

\section{References}

Baker, E. J., \& Alluisi, E. A. Information handling aspects of visual and auditory form perception. J. engng Psychol., 1962, 1, 159-179.

Fitts, P. M., \& Leonard, J. A. Stimulus correlates of visual pattern recognition-a probability approach. Wash., D. C., Off. Naval Res., Final Rep. of Contract Nonr-495(02), October, 1957.

Fitts, P. M., \& Switzer, G. Cognitive aspects of information processing: I. The familiarity of S-R sets and subsets. J. exp. Psychol., 1962, 63, 321-329.

Michels, K. M., \& Zusne, L. Metrics of visual form. Psychol. Bull., $1965,63,74-86$.

\section{Note}

1. Supported in part by the U. S. Army Medical Research and Development Command, Department of the Army, under Research Contract No. DA-49-193-MD-2567, “Behavioral Effects of Infectious Diseases." 